\section{Sleeping Beauty gets specific}

\section{By Kai-Jye Lou, Staff Writer}

Two international groups of researchers have expanded the utility of a transposon-based mutagenesis system, dubbed Sleeping Beauty, by adding tissue specificity to the system. They are now able to induce a wider range of cancers compared with the capacity of earlier iterations of the technology. ${ }^{1,2}$ The increased reach of the technology has already allowed the groups to identify new disease-associated genes in hepatocellular carcinoma and colorectal cancer.

The Sleeping Beauty transposon system contains two parts: a transposase enzyme and the transposon vector. In transgenic Sleeping Beauty model organisms, activation of the enzyme causes the transposon to induce traceable, random insertional mutations in host somatic cell genomes. The mutations can be identified with high throughput sequencing.

Earlier iterations of the technology employed a universal promoter for transposase expression, which caused insertional mutations to occur in all tissues. Mice generated using the original system primarily developed hematopoietic tumors and only a few solid tumor types such as soft-tissue sarcomas, pulmonary adenocarcinomas and malignant meningiomas. ${ }^{3}$ Also, the inability to generate tumors in a tissue-specific manner made it difficult to track the origins of a particular cancer cell.

To overcome these shortcomings, overlapping research groups led by David Largaespada integrated an additional element of control into the platform: a conditional, tissue-specific promoter that restricts Sleeping Beauty transposase expression. Largaespada is leader of the genetic mechanisms of cancer research program at the University of Minnesota's Masonic Cancer Center.

In a paper in Nature Biotechnology, one group reported that they put transposase expression under the control of a hepatocyte-specific conditional promoter to induce hepatocellular carcinoma (HCC). In an article in Science, the other group reported their use of a gastrointestinal epithelial cell-specific conditional promoter to induce colorectal cancer.

Each group of researchers then used a mouse genetics screen. The Nature Biotechnology team found 15 genes and the Science team found 17 genes whose associations had not been previously reported in HCC and colorectal cancer, respectively.

"Results from these papers expand our understanding of the genes involved in colorectal and liver cancer, providing insight into the nature of these diseases and ultimately providing diagnostic and therapeutic tools," said R. Scott McIvor, a professor in the Department of Genetics, Cell Biology and Development at the University of Minnesota.

McIvor is also CEO and CSO at Discovery Genomics Inc., which is developing the Sleeping Beauty system for use in human gene therapy. "The greater understanding of the group of genes contributing to colorectal and liver cancer will eventually lead to the development of improved therapies for these diseases," he said.

"The important thing here is that they've been able to limit the activity of the Sleeping Beauty transposon to specific organs and tissues," said Perry Hackett, a professor in the university's Department of Genetics, Cell Biology and Development and who is the original developer of the Sleeping Beauty transposon system. ${ }^{4}$

"This conceptual breakthrough allows one to study a broader range of cancers, including slowly developing tumors," Hackett added.

Results from a previous paper by Largaespada and a team at the National Cancer Institute showed that an earlier iteration of the Sleeping Beauty system caused mice to rapidly succumb to hematopoietic tumors before slow-growing solid tumors had the opportunity to develop.

Tissue-specific induction also makes it possible to study the origins and progression of a cancer. "By limiting the tissue of origin where the transposase is activated, they are able to separate out original tumors from metastasis," Hackett told SciBX. "In the Nature Biotechnology paper, they were able to show that tumors appearing in the lung originated from HCC cells in the liver."

"Taken together, these results show that the Sleeping Beauty transposon system can be used as a generally applicable tool for identifying new targets for cancer treatment, detection and prognosis," Largaespada told SciBX. "The Sleeping Beauty transposon system also can aid in the identification of major nodes in cancer pathways and provide insights on what combination therapies to use to target them."

Anton Berns, director of research at the Netherlands Cancer Institute, said the data generated from Sleeping Beauty-based insertional mutagenesis studies may help identify combinations of interdependent mutations, thus giving additional hints at which combinations of genes to target.

\section{More work ahead}

With the two proof-of-concept studies from Largaespada's group supporting use of the Sleeping Beauty system in cancer gene discovery, researchers contacted by SciBX think the system will now be better positioned to complement other genomics systems and gene association studies.

"Transposons can only do certain things-they can only insert," Berns noted. Transposons can activate or inactivate a gene as a result but are unable to induce point mutations, which are often seen in human tumors, he said. 
For example, Berns said, the Sleeping Beauty system cannot generate activating point mutations in genes like Ras, which are known to be strong drivers of cancer in humans. "There is a limit to the types of activating mutations that can be generated," he concluded.

Berns said follow-up gene ablation or overexpression studies on the identified cancer-associated genes also will be needed to validate their roles in cancer.

Largaespada said his group is using Sleeping Beauty to identify additional cancer-associated genes and study other forms of the disease, including brain and lung cancer. He added that he would also like to develop a Sleeping Beauty-based screening system to study cancer phenotypes and identify genes associated with metastasis, recurrence and drug resistance.

Largaespada told SciBX that a patent covering the use of the Sleeping Beauty transposon system to identify cancer genes in laboratory animals is pending. The technology is available for licensing from the Office for Technology Commercialization at the University of Minnesota.
Lou, K.-J. SciBX 2(11); doi:10.1038/scibx.2009.433

Published online March 19, 2009

\section{REFERENCES}

1. Keng, V.W. et al. Nat. Biotechnol.; published online Feb. 22, 2009; doi:10.1038/nbt.1526

Contact: David Largaespada, University of Minnesota, Minneapolis, Minn. e-mail: larga002@umn.edu

2. Starr, T.K. et al. Science; published online Feb. 26, 2009; doi:10.1126/science. 1163040

Contact: David Largaespada, University of Minnesota, Minneapolis, Minn. e-mail: larga002@umn.edu

Contact: Timothy K. Starr, same affiliation as above e-mail: star0044@umn.edu

3. Collier, L.S. et al. Nature 436, 272-276 (2005)

4. Ivics, Z. et al. Cell 91, 501-510 (1997)

5. Dupuy, A.J. et al. Nature 436, 221-226 (2005)

COMPANIES AND INSTITUTIONS MENTIONED

Discovery Genomics Inc., Minneapolis, Minn.

National Cancer Institute, Bethesda, Md.

Netherlands Cancer Institute, Amsterdam, the Netherlands University of Minnesota, Minneapolis, Minn. 